\title{
Wide-Field Optical Coherence Tomography Angiography for Early Detection and Objective Evaluation of Proliferative Diabetic Retinopathy
}

Hagar Khalid, ${ }^{* 1,2}$ Roy Schwartz, ${ }^{* 1}$ Luke Nicholson, ${ }^{1}$ Josef Huemer, ${ }^{1,3}$ Mohamed El-Bradey, ${ }^{2}$ Dawn A. Sim, ${ }^{1}$ Praveen J. Patel, ${ }^{1}$ Konstantinos Balaskas, ${ }^{1}$ Robin Hamilton, ${ }^{1}$ Pearse A. Keane, ${ }^{1}$ Ranjan Rajendram ${ }^{1}$

${ }^{1}$ NIHR Biomedical Research Centre at Moorfields Eye Hospital NHS Foundation Trust and UCL Institute of Ophthalmology, London, UK.

2 Ophthalmology Department, Faculty of Medicine, Tanta University, Tanta, Egypt

${ }^{3}$ Eye Department, Tauernklinikum, Zell am See, Austria

*Hagar Khalid and Roy Schwartz contributed equally to the submission.

\section{Correspondence and reprint requests:}

Hagar Khalid, MRCSEd, NIHR Biomedical Research Centre for Ophthalmology, Moorfields Eye Hospital NHS Foundation Trust and UCL Institute of Ophthalmology, United Kingdom. Tel: +44 7404229463 Email: hagar.khalid@nhs.net

\section{Keywords:}

Proliferative diabetic retinopathy

Grading

OCT

OCTA

Wide-field

Neovascularization 


\title{
SYNOPSIS:
}

Using wide-field optical coherence tomography angiography (OCTA) resulted in higher detection rate of proliferative diabetic retinopathy than clinically. Phenotypic characterization of neovascularization at the disc on OCTA is also provided.

\begin{abstract}
Purpose: To evaluate the utility of wide field optical coherence tomography angiography (WF-OCTA) compared to clinical examination in grading diabetic retinopathy in patients suspected clinically to have proliferative diabetic retinopathy (PDR).

Design: This retrospective observational case series included patients diagnosed clinically with PDR or severe non-proliferative diabetic retinopathy (NPDR). Patients underwent standard clinical examination and wide-field OCTA Imaging (PLEX Elite 9000, Carl Zeiss Meditec, AG) using 12×12 montage scans between August 2018 and January 2019. Two trained graders identified neovascularisation at the disc (NVD) and elsewhere (NVE) on OCTA which were compared to the clinical examination, and to ultrawide-field fluorescein angiography (UWF-FFA) when available.

Results: Seventy-nine eyes of 46 patients were evaluated. Of those, 57 eyes were diagnosed clinically with PDR, and 22 with severe NPDR. NVD was detected on OCTA-B scan as PRHM in 39 eyes $(100 \%)$ with evident flow signals in $79.5 \%$ compared to $51.3 \%$ detected clinically. We further classified NVD on OCTA into four subtypes and found that subtypes 1 and 2 could not be seen on clinical examination alone. OCTA detected NVE in $81 \%$ of the cases compared to $55.7 \%$ detected clinically. Using WF-OCTA resulted in a higher percentage of PDR grading (88.6\%) than on clinical examination $(72.2 \%)$. When available, FFA confirmed the OCTA diagnosis in the majority of cases.

Conclusion: This study demonstrates that WF-OCTA may be a more sensitive tool in diagnosing PDR than clinical examination, while providing the same rate of diagnosis as FFA or more. This suggests that this modality has the potential to replace FFA non-invasively for the purpose of detection and characterisation of neovascularisation.
\end{abstract}




\section{INTRODUCTION}

Proliferative diabetic retinopathy (PDR) is the advanced stage of diabetic retinopathy (DR) and is characterized by the development of abnormal blood vessels (neovascularisation), which may be of the disc (NVD) or elsewhere (NVE), secondary to retinal ischaemia.(1) If left untreated, neovascularisation may lead to haemorrhagic and tractional complications which may be vision-threatening. (1),(2) Therefore, early detection of PDR is essential to facilitate early intervention

The Early Treatment Diabetic Retinopathy Study (ETDRS) established the current standard method of assessing the severity of DR. It is based on seven standard retinal fields seen on stereoscopic color fundus photographs (CFPs). ${ }^{(3)}$ However, the small retinal field covered and its dependence on CFP limits its ongoing application in patients with DR. Recent advances in retinal vascular imaging provide more details on diabetic retinal changes compared to standard CFPs. ${ }^{(4),(5)}$ Conventional fundus cameras cover a wider field of 45 to 60 degrees in one exposure. Furthermore, new generations of high-resolution ultrawide field (UWF) cameras can capture 130 degrees or even 200 degrees in a single image, permitting evaluation of peripheral retinal lesions that are only observed to a limited extent or missed completely in the area typically encompassed by seven standard fields. ${ }^{(6)}$ Similarly, UWF fundus fluorescein angiography (UWF-FFA) can acquire high-resolution, widefield angiographic data in a single 100- or 200-degree frame which improves visualisation of key peripheral retinal pathology. ${ }^{(6),(7)}$ This may result in a more severe grading of DR, and thus affect patient management.(8)-(11)

Recently, The ability to clearly visualize microvascular changes in DR in a noninvasive, safe and easily repeatable manner has become possible with the introduction of OCT angiography (OCTA), which allows for the assessment of retinal ischaemia and neovascularisation.(4),(12) OCTA may potentially facilitate staging of DR or even detection of early DR changes prior to clinically detectable retinopathy. ${ }^{(13)}$ Thus, it shows promise in supplementing clinical examination by potentially replacing FFA for the diagnosis and monitoring of DR. Until recently, this was not achievable due to the limited field of view of earlier devices. However, a swept-source OCTA (SS-OCTA) device has become available, allowing for montaging of several scans in real-time, thus providing almost 80 degrees field of view in a single scan. (2),(14),(15)

The purpose of this study is to compare clinical examination and UWFFFA, when available, with WF-OCTA in grading DR in patients suspected to have PDR by clinical examination

\section{METHODS}

\section{Data Collection:}

This was a retrospective observational case series. It included patients diagnosed clinically with PDR (either naïve or previously treated), or severe non-proliferative DR in whom early PDR was suspected without clinically evident neovascularization (due to unexplained vitreous haemorrhage, PDR in the other eye, or inconclusive differentiation between intraretinal 
microvascular abnormalities (IRMA) and NVE). Patients underwent SS-OCTA Imaging (PLEX Elite 9000, Carl Zeiss Meditec,AG) as part of routine clinical care between August 2018 and January 2019.

PDR was diagnosed clinically by the detection of presumed retinal neovascularisation, and/or preretinal or vitreous haemorrhage. Stable PDR was defined when preretinal fibrosis and peripheral scatter laser could be detected. Severe NPDR was diagnosed if there were diffuse intraretinal hemorrhages and microaneurysms in four quadrants, venous beading in two quadrants, or IRMA in one quadrant, based on the ETDRS classification. ${ }^{(16)}$ Eyes were excluded if they had severe media opacities preventing a clear scan, or if the image quality was otherwise judged insufficient to allow clinical assessment.

Demographic and clinical data were collected for all patients. Approval for data collection and analysis was obtained from the Institutional Review Board at Moorfields (ROAD17/034). The study adhered to the tenets set forth in the Declaration of Helsinki.

\section{Image Acquisition Protocol:}

OCTA scans were obtained using the PLEX Elite 9000. It uses a sweptsource tunable laser with wavelength of $1060 \mathrm{~nm}$ and with a scanning rate of 100,000 A-scans per second, providing a transverse resolution of $20 \mu \mathrm{m}$ and an in-depth resolution (digital) of $1.95 \mu \mathrm{m}$. This instrument has an A-scan depth of $3.0 \mathrm{~mm}$ in tissue (1536 pixels). The $12 \times 12 \mathrm{~mm}$ scans used $500 \mathrm{~A}$ scans per B-scan at 500 B-scan positions, resulting in an A-scan and B-scan separation of $24 \mu \mathrm{m}$. Two sequential B-scans were performed at each fixed position before proceeding to the next transverse location on the retina.

The scanning protocol used was a $12 \times 12 \mathrm{~mm}$ OCTA Montage, in which five $12 \times 12 \mathrm{~mm}$ OCTA data cubes positioned at predefined locations (central, superotemporal, superonasal, inferotemporal, and inferonasal) were acquired. WF-OCTA en face montage was automatically generated with a total field of view up to $80^{\circ}$ (Figure 1 ).

The en face OCTA images visualized the flow that was detectable within a volume defined by selected boundaries. Each of these segmented volumes is referred to as a slab. To detect neovascularization, the vitreoretinal interface (VRI) segmentation and superficial retinal segmentation were used. The superficial retinal slab was defined as the volume between the internal limiting membrane (ILM) and the outer boundary of the inner plexiform layer (IPL). The vitreoretinal interface (VRI) slab was defined with an inner boundary $200 \mu \mathrm{m}$ above the ILM and an outer boundary positioned on the ILM. Some VRI slabs were corrected manually to ensure that the entire neovascular complex was contained within the slab, especially in cases of extensive neovascularisation

FFA photographs were acquired using the ultra-widefield Optos 200TX (Optos Plc, Dunfermline, Scotland) in selected cases. 


\section{Image Grading:}

Two masked, trained retina specialists (H.K., L.N.) independently reviewed structural OCT B-scans with overlay flow signal, and the en face OCTA images of the superficial capillary plexus and VRI in each cube of the OCTA montage, as well as on FFA. Each grader assessed the degree of DR based on OCTA scans, and on FFA images when available. This evaluation was then compared with the clinical grading given at the time of clinical examination.

Neovascularization of the disc (NVD) and elsewhere (NVE) were identified as preretinal hyperreflective material (PRHM) on OCT B-scans. The finding of a flow signal within the PRHM was recorded, designating activity of the neovascularisation. This was correlated with OCTA en face images, where the finding of any abnormal vascular network at the level of the corresponding B-scan with PRHM was recorded (Figure 2).

In order to further distinguish between clinical examination and OCTA we sub classified NVD into four types according to its configuration on OCTA: Type 1 - in which NVD "bridges" the physiological cup, and which could be considered the earliest stage; Type 2 - in which NVD is seen as small buds; Type 3 - where the NVD is seen flat over the ILM; and Type 4 - in which the NVD is elevated into the vitreous (Figure 3).

IRMA were differentiated from NVE based on penetration of the ILM by the latter (figure 4).

In selected cases where UWF-FFA was done, the finding of leakage in areas suspected to be neovascularisation by clinical examination and OCT was recorded.

Disagreements between graders were adjudicated by a senior retina specialist (R.R.).

\section{Statistical analysis}

Inter-rater agreement was calculated using kappa statistics, wherein a Kappa (K) value of less than 0.2 means poor agreement, 0.21-0.40 fair agreement, 0.41-0.60 moderate agreement, 0.61-0.80 good agreement, and 0.81-1.00 very good agreement. (17) Statistical analyses were performed using MedCalc version 19.0.5 (MedCalc Inc., Mariakerke, Belgium).

\section{RESULTS}

A total of 79 eyes of 46 patients were included in the study. Of those, 57 eyes were diagnosed clinically as PDR (43 eyes with active PDR and 14 eyes with stable PDR), and 22 eyes were suspected to have early PDR without evident neovascularisation clinically. Of PDR cases 32 eyes were naïve and 47 were previously treated. The inter-rater agreement was 0.83 for NVD detection on OCTA, 0.89 for NVE detection on OCTA, 1.0 for structural OCT, and 1.0 for FFA. 


\section{Detection of Retinal NVD}

Preretinal hyperreflective material (PRHM) was detected in 39 eyes of 28 patients $(100 \%)$ on B-scan OCT, with a flow signal detected in $31 / 39$ eyes (79.5\%). En face OCTA detected neovascularisation in $28(71.8 \%)$ eyes, all of them with a flow signal on the B-scan. Of undetectable cases on en face OCTA, eight did not have flow signals on the B-scan and in three eyes the flow was minimal. On clinical examination NVD was detected in 20 eyes (51.3\%). Eleven eyes had same-visit fluorescein angiography which could confirm the presence of NVD in five eyes (45.5\%), was inconclusive in two eyes, and could not detect NVD in the remaining four eyes (Table 1).

\section{Classification of NVD according to OCTA characteristics}

To further elucidate the discrepancy between the clinical exam and OCTA in diagnosis of NVD, we classified NVDs into four types and compared the rate of detection by each modality. In some cases, more than one type could be detected in each eye. Twenty-six eyes had a Type 1 NVD, six eyes Type 2, 19 eyes Type 3, and ten eyes Type 4 (Table 1 ).

Type 1 NVDs were usually detected in the superficial retinal plexus slab, which encompasses the physiological cup in the segmentation. Other types were best detected in the VRI segmentation slab. All eyes with a Type 4 NVD were detected clinically, while Type 1 or Type 2 could not be detected clinically, and Type 3 could be detected in some cases (Figure 3 ).

\section{Detection of Retinal NVE}

For the purpose of NVE detection, 383 OCT cubes of 79 eyes were analysed. NVE were detected on B-scan OCT in 64/79 eyes (81\%). A flow signal was detected in $62 / 79$ eyes $(78.5 \%)$. Similarly, en face OCTA was able to detect NVE in $62 / 79$ eyes (78.5\%) ranging from only one cube involved to widely distributed NVEs in all five cubes. On examination, NVE was detected in 44/79 (55.7\%) eyes.

Of clinically diagnosed stable cases, where preretinal fibrosis and peripheral scatter laser could be detected, NVE with evident flow in OCTA B-scan still could be seen in 10/14 eyes (71.4\%). Twenty-four eyes had UWF-FFA which confirmed the presence of NVE in nine eyes (37.5\%) and was inconclusive in one eye, compared with 15 of 24 eyes $(62.5 \%)$ where NVE was detected by OCTA (Table 1). Of the five undetectable cases on FFA, one case did not show flow signals on B-scan OCTA and the others were small with a mean elevation of 103.5 microns (range:70-127 microns) and mean horizontal diameter 313.75 microns (range: 162-521), as measured using the built-in caliper in the OCT device. Additionally, all of the four undetectable NVEs originated from large retinal vessels (Figure 5). In two eyes, FFA detected more NVEs, which were beyond the field of view of the OCTA scan. 


\section{Grading of Diabetic Retinopathy}

Analysis of OCTA scans resulted in a diagnosis of PDR in 70 eyes in comparison with 57 eyes when the diagnosis was based on clinical examination. NPDR was diagnosed in in nine eyes by OCTA vs. 22 on clinical examination. (Table 1). Amongst clinically diagnosed PDR, evidence of neovascularisation could be detected on OCTA in all cases (100\%). Of the 22 eyes diagnosed clinically with severe NPDR,13 eyes (59\%) showed neovascularisation on OCTA. Of those 13 eyes, nine had an NVE that was very fine and was overlooked or possibly misdiagnosed as a retinal haemorrhage. In seven eyes NVDs were missed on clinical examination alone.

Of the 24 patients who had both WF-OCTA and UWF-FFA, there was an agreement in diabetic retinopathy grading in 21 eyes (87.5\%) (six eyes with severe NPDR and 15 eyes with PDR) In three eyes neovascularization could be detected on OCTA but not on FFA. As previously mentioned, in two eyes, FFA detected more NVEs than OCTA, which were beyond the field of view of the OCTA scan. However, this did not result in a change in the grading of diabetic retinopathy.

\section{DISCUSSION:}

In this study we attempted to compare the detection of neovascularisation, and thereby grading of DR, on OCTA versus clinical examination carried out in real world clinics in patients diagnosed or suspected to have PDR clinically. We found that WF-OCTA detected more neovascularisation than was clinically obvious, resulting in a higher percentage of PDR detection. For the detection of NVD, we found that certain OCTA subtypes (Type 1 and Type 2) could not be seen on clinical examination alone. When available, FFA confirmed the OCTA diagnosis in the majority of cases (87.5\%), implying that the findings on OCTA were accurate. These findings suggest that WF-OCTA could be considered in the daily management of DR, particularly in those patients with severe non-proliferative DR on clinical examination or retinal photography.

The highest rate of detection of NVDs was on OCT (100\%), as expected given its highest detection rate, as we have previously shown.(18) This was followed by B-scan OCTA $(79.5 \%)$, en face OCTA $(71,8 \%)$, and clinical examination (51.3\%). Interestingly, the different NVD anatomical subtypes detected on OCTA can explain the lower detection rate on fundoscopy, as we found that most of the clinically undetectable NVD resulted from subtypes 1 and 2. A possible explanation is that type 1 NVDs encompass the physiological cup and are therefore not elevated above the disc surface, making it hard to detect them clinically. Similarly, type 2 NVDs, which appear on the B-scan as tiny buds, may be misdiagnosed clinically as dots of haemorrhage. To the best of our knowledge, this is the first observation of different configurations of NVD on OCTA with a clinical implication. All cases identified using en face OCTA were also positive on B- 
scan OCTA, but the opposite was not true. En face OCTA is prone to various artifacts, which accounted for some of the misdetections of neovascularisation in our cohort, which signifies the need to inspect the accompanying B-scan flow signal.

NVEs were mostly detected on B-scan OCT, with a detection rate of $81 \%$, followed by B-scan OCTA and en face OCTA $(78.5 \%)$, and a detection rate of only $55.7 \%$ on clinical examination. Different factors may explain the lower detection rate on clinical examination alone. First, the presence of haemorrhage may mask details of neovascularisation and small NVEs could be misdiagnosed as preretinal haemorrhages. Second, some vessels may be too fine to detect, particularly against the background of a normal retinal vessel, without the use of high-resolution imaging. Additionally, the distinction between an IRMA and neovascularization, which is a common clinical dilemma, may be difficult without the assistance of OCT B-scan to show the exact location of the vessel in relation to the ILM.

Few studies described the detection and characteristics of NVE by OCTA, and to a lesser extent the detection of NVD.(1),(19)-(21). Schaal et al assessed the detection of vascular features in diabetic patients using WFOCTA in comparison with CFPs. Similar to our findings on clinical examination, they found that OCTA was more sensitive in the detection of neovascularisation, as it could detect neovascularisation not seen on CFP in $12 \%$ of cases. In a study by You et al. utilising a different WF-OCTA protocol, which included only patients diagnosed clinically with NPDR, any flow signal on the VRI slab was considered to be neovascularisation, which was detected by OCTA in four eyes of the 27 included participants. According to the authors, clinical examination may have missed these vessels due to previous treatment with anti-VEGF, which may have altered their visibility. ${ }^{(13)}$ In our cohort, none of the patients diagnosed clinically with NPDR were previously treated with anti-VEGF. It is still unclear whether the sensitivity of OCTA in detecting small neovascularisation not visible on clinical examination has clinical significance and if this neovascularization should be treated. Further longitudinal studies are required to compare the complication rates of PDR based on treatment decisions made on multimodal imaging versus current practice

In terms of DR grading, we found that detection of neovascularisation based on WF-OCTA resulted in $88.6 \%$ of cases diagnosed with PDR in comparison with only $72.2 \%$ on clinical examination. Our results are similar to the previously mentioned study by Schaal et al, in which an additional $12 \%$ of participants were found to have PDR when SS-OCTA was used in comparison with CFP alone. ${ }^{(4)}$

Of eyes that had both WF-OCTA and WF-FFA on the same visit, all neovascularisations that were detected on OCTA could be detected on FFA. In two cases FFA detected more NVEs than OCTA. However, they were outside the field of view of the WF-OCTA. This is consistent with prior studies that have shown the majority of neovascularisation in PDR were located in the 
posterior pole. (22)-(24) Meanwhile, FFA could not detect neovascularisation that was detected in WF-OCTA in $12.5 \%$ of the eyes. This could be explained by the ability of OCTA to detect subtle flow within the vessels and better characterization of the neovascularization in OCTA as there is no vascular leakage obscuring the vascular details. Additionally, it could be explained by the size of neovascularization. There may be a threshold for the detection of neovascularisation by FFA, wherein below a certain size neovascularization might not leak or could be misdiagnosed as a microaneurysm or a different existing vascular structure. In our cohort, the mean elevation of undetected neovascularisation was 103.5 microns and the mean diameter was 313.75 microns. More studies are needed to differentiate the detection rate of neovascularisation by FFA based on size in comparison with OCTA.

Our study has several limitations. First, the retrospective nature of the study. Second, FFA was not done for every patient, as is customary in realworld clinic setting. This necessitated OCTA to be compared with clinical examination, which may be subjective. However, each of the cases was seen by an experienced retinal physician who confirmed the diagnosis. Finally, as discussed, although the OCTA device used in this study provides wide-field images, the field of view is still smaller than that provided by UWF-FFA. However, based on previous studies (22)-(24)and our findings, most of the neovascularization in PDR are mid peripheral, and in our cohort this did not result in a misdiagnosis of the DR grade

In summary, our findings suggest that OCTA may be a more sensitive tool in the diagnosis of proliferative DR than clinical examination, while providing the same rate of detection as FFA or more. Furthermore, OCTA highlighted that detailed phenotypic characterisation of neovascularisation is possible, and this may have prognostic implications impacting upon clinical practice in the future

\section{ACKNOWLEDGMENTS/DISCLOSURE:}

a. Funding/Support: Dr Khalid supported by a scholarship from Egyptian mission sector, Ministry of Higher Education, Egypt .

b. Financial disclosures: Mr Rajendram has received speaker fees from Zeiss. Dr. Keane has received speaker fees from Heidelberg Engineering, Topcon, Carl Zeiss Meditec, Haag-Streit, Allergan, Novartis, and Bayer. He has served on advisory boards for Novartis and Bayer and has been an external consultant for DeepMind and Optos. Dr. Keane is supported by a United Kingdom (UK) National Institute for Health Research (NIHR) Clinician Scientist Award (NIHR-CS--2014-12-023). Dr Schwartz has received travel expenses from Allergan. Dr Nicholson has received speaker fees from Allergan. Other authors have no financial disclosures."

c. Acknowledgments: Hagar Khalid and Roy Schwartz equally contributed to the submission, NIHR Biomedical Research Centre at Moorfields Eye Hospital NHS Foundation Trust and UCL Institute of Ophthalmology, London, UK. 


\section{REFERENCES:}

1. de Carlo TE, Bonini Filho MA, Baumal CR, Reichel E, Rogers A, Witkin AJ, et al. Evaluation of Preretinal Neovascularization in Proliferative Diabetic Retinopathy Using Optical Coherence Tomography Angiography. Ophthalmic Surgery, Lasers Imaging Retin [Internet]. 2016;47(2):115-9. Available from:

http://www.healio.com/doiresolver?doi=10.3928/23258160-2016012603

2. Bandello F, Corbelli E, Carnevali A, Pierro L, Querques G. Optical Coherence Tomography Angiography of Diabetic Retinopathy. Dev Ophthalmol. 2016;56:107-12.

3. Treatment E, Retinopathy D. Fundus Photographic Risk Factors for Progression of Diabetic Retinopathy: ETDRS Report Number 12. Ophthalmology [Internet]. 1991;98(5):823-33. Available from: http://dx.doi.org/10.1016/S0161-6420(13)38014-2

4. Schaal KB, Munk MR, Wyssmueller I, Berger LE, Zinkernagel MS, Wolf S. Vascular Abnormalities in Diabetic Retinopathy Assessed With Swept-Source Optical Coherence Tomography Angiography Widefield Imaging. Retina [Internet]. 2017;1. Available from: http://insights.ovid.com/crossref?an=00006982-900000000-96708

5. Lee J, Rosen R. Optical Coherence Tomography Angiography in Diabetes. Curr Diab Rep [Internet]. 2016;1980. Available from: http://dx.doi.org/10.1007/s11892-016-0811-x

6. Wessel MM, Aaker GD, Parlitsis G, Cho M, Amico DJD. Improves the Detection and Classification of Diabetic Retinopathy. Retina. 2012;32:785-91.

7. Silva PS, Cavallerano JD, Tolls D, Omar A, Thakore K, Patel B, et al. Potential efficiency benefits of nonmydriatic ultrawide field retinal imaging in an ocular telehealth diabetic retinopathy program. Diabetes Care. 2014;37(1):50-5.

8. Silva PS, Cavallerano JD, Sun JK, Soliman AZ, Aiello LM, Aiello LP. Peripheral lesions identified by mydriatic ultrawide field imaging: Distribution and potential impact on diabetic retinopathy severity. Ophthalmology [Internet]. 2013;120(12):2587-95. Available from: http://dx.doi.org/10.1016/j.ophtha.2013.05.004

9. Kimble JA, Brandt BM, Mcgwin G. Locates Capillary Nonperfusion in Diabetic Retinopathy. :555-7.

10. Oliver SCN, Schwartz SD. Peripheral Vessel Leakage (PVL): A new angiographic finding in diabetic retinopathy identified with ultra widefield fluorescein angiography. Semin Ophthalmol. 2010;25(1-2):27-33.

11. Soliman AZ, Silva PS, Aiello LP, Sun JK. Ultra-wide field retinal imaging 
in detection, classification, and management of diabetic retinopathy. Semin Ophthalmol. 2012;27(5-6):226-32.

12. Choi W, Waheed NK, Moult EM, Adhi M, Duker JS, Fujimoto JG. Ultrahigh Speed OCT Angiography of Retinal and Choriocapillaris Alterations in Diabetic Patients with and without Retinopathy Using Swept Source Optical Coherence Tomography WooJhon. 2018;37(May 2014):11-21.

13. You QS, Guo Y, Wang J, Wei X, Camino A, Zang P, et al. Detection of Clinically Unsuspected Retinal Neovascularization With Wide-Field Optical Coherence Tomography Angiography. Retina. 2019;1.

14. Baumal CR, Adhi M, Witkin AJ, Moult E, Waheed NK, Salz DA, et al. Select Features of Diabetic Retinopathy on Swept-Source Optical Coherence Tomographic Angiography Compared With Fluorescein Angiography and Normal Eyes. JAMA Ophthalmol. 2016;134(6):644.

15. Ishibazawa A, Nagaoka T, Takahashi A, Omae T, Tani T, Sogawa K, et al. Optical coherence tomography angiography in diabetic retinopathy: A prospective pilot study. Am J Ophthalmol [Internet]. 2015;160(1):3544.e1. Available from: http://dx.doi.org/10.1016/j.ajo.2015.04.021

16. Wilkinson CP, Ferris FL, Klein RE, Lee PP, Agardh CD, Davis M, et al. Proposed International Clinical Diabetic Retinopathy and Diabetic Macular Edema Disease Severity Scales. Ophthalmology [Internet]. 2003 [cited 2019 Apr 6];110:1677-82.

17. Brennan $P$, Silman A. Statistical methods for assessing observer variability in clinical measures. BMJ [Internet]. 1992 Jun 6 [cited 2019 Sep 28];304(6840):1491-4. Available from:

http://www.bmj.com/cgi/doi/10.1136/bmj.304.6840.1491

18. Schwartz R, Khalid H, Sivaprasad S, Nicholson L, Anikina E, Sullivan P, et al. Objective evaluation of proliferative diabetic retinopathy using optical coherence tomography. Ophthalmol Retin. 2019 Sep;

19. Ishibazawa A, Nagaoka T, Yokota H, Takahashi A, Omae T, Song YS, et al. Characteristics of retinal neovascularization in proliferative diabetic retinopathy imaged by optical coherence tomography angiography. Investig Ophthalmol Vis Sci. 2016;57(14):6247-55.

20. Pan J, Chen D, Yang X, Zou R, Zhao K, Cheng D, et al. Characteristics of Neovascularization in Early Stages of Proliferative Diabetic Retinopathy by Optical Coherence Tomography Angiography. Am J Ophthalmol [Internet]. 2018;192:146-56. Available from: https://doi.org/10.1016/j.ajo.2018.05.018

21. Hirano T, Kakihara S, Toriyama Y, Nittala MG, Murata T, Sadda S. Wide-field en face swept-source optical coherence tomography angiography using extended field imaging in diabetic retinopathy. $\mathrm{Br} \mathrm{J}$ 
Ophthalmol. 2018;102(9):1199-203.

22. Feman SS, Leonard-Martin TC, Semchyshyn TM. The Topographic Distribution of the First Sites of Diabetic Retinal Neovascularization. Am J Ophthalmol [Internet]. 1998 May 1 [cited 2019 May 20];125(5):704-6. Available from:

https://www.sciencedirect.com/science/article/pii/S0002939498000130

23. Jansson RW, Froystein T, Krohn J. Topographical distribution of retinal and optic disc neovascularization in early stages of proliferative diabetic retinopathy. Investig Ophthalmol Vis Sci. 2012;53(13):8246-52.

24. Taylor E, Dobree JH. Proliferative diabetic retinopathy. Site and size of initial lesions. Br J Ophthalmol. 1970;54(1):11-8. 


\section{FIGURE LEGENDS}

\section{Figure 1}

a) UltraWide field fluorescein angiography (UWF-FFA) with overlying dotted blue square of the corresponding wide-field optical coherence tomography angiography (WF-OCTA) field, illustrating that despite the wider field of UWFFFA, most of clinically significant data was detected within the WF-OCTA field. Additionally, the peripheral field in UWF-FFA shows artifacts that affect image interpretation. b) widefield OCTA montage with overlaid squares demonstrating the different retinal sectors imaged by the $5(12 \times 12)$ data cubes; Central, superonasal, superotemporal, inferonasal, and inferotemporal cubes.

\section{Figure 2}

Wide-field en face optical coherence tomography angiography (WF-OCTA) montage at the level of the superficial capillary plexus slab showing multiple neovascularisation elsewhere (NVEs) mid peripherally (red arrow) at the edge of the ischaemic areas (star). Neovascularization of the disc (NVD) could also be detected (yellow arrow)

\section{Figure 3}

En face optical coherence tomography angiography (OCTA) at the superficial and vitreoretinal interface (VRI) slabs and the corresponding OCTA B-scans to illustrate the different types of neovascularization of the disc (NVD): a) Type 1 NVD: OCTA B-scan demonstrating demonstrating a preretinal hyperreflective material (PRHM) bridging the physiological cup with evident flow signal which could be seen at the superficial slab as it encompasses the physiological cup within. b) Type 2 NVD: which appear as small buds in the Bscan which could be seen at the VRI slab. c)Type 3 NVD: where the PRHM can be seen in the B-scan flat over the surface. This type could be detected in the VRI slab. d)Type 4 NVD: which is elevated above the surface as seen in the B-scan. This type is detectable at the VRI slab.

\section{Figure 4:}

Central 12x12 enface optical coherence tomography Angiography (OCTA) at the superficial $(A)$ and vitreoretinal interface (VRI) $(B)$ slabs with multiple OCTA B-scans at different levels illustrating various diabetic retinopathy signs.

C) Neovascularisation elsewhere (NVE) could be prominently seen as a preretinal hyperreflective material (PRHM) in the B-scan with evident flow signals which could be detected as network at the VRI level (yellow star), while intraretinal microvascular abnormalities (IRMA) (white arrow head) could be seen on the enface OCTA at the superficial slab as abnormal dilated blood vessels without any evident PRHM on the B-scan (E). OCTA may be helpful in differentiating PRHM that is secondary to preretinal haemorrhage (red arrow head) from an NVE (yellow star). Preretinal haemorrhage characterised by the absence of flow signals on OCTA B-scan along with backshadowing (D) OCTA can detect vascular loops as well (blue arrow) (F)

\section{Figure 5:}


a) Late phase fluorescein angiography of the Inferotemporal quadrant showing small hyperfluorescent lesion (red arrow) that were diagnosed as microaneurysms. However, the corresponding enface OCTA (b) illustrated the presence of a small NVE (red arrow) over the infero-temporal arcade corresponding to preretinal hyperreflective material (PRHM) with a flow signal on OCTA B-scan (with vertical diameter of $127 \mu \mathrm{m}$ and horizontal diameter of $203 \mu \mathrm{m})$ 
Table 1: NVD and NVE detection rate using different image modalities

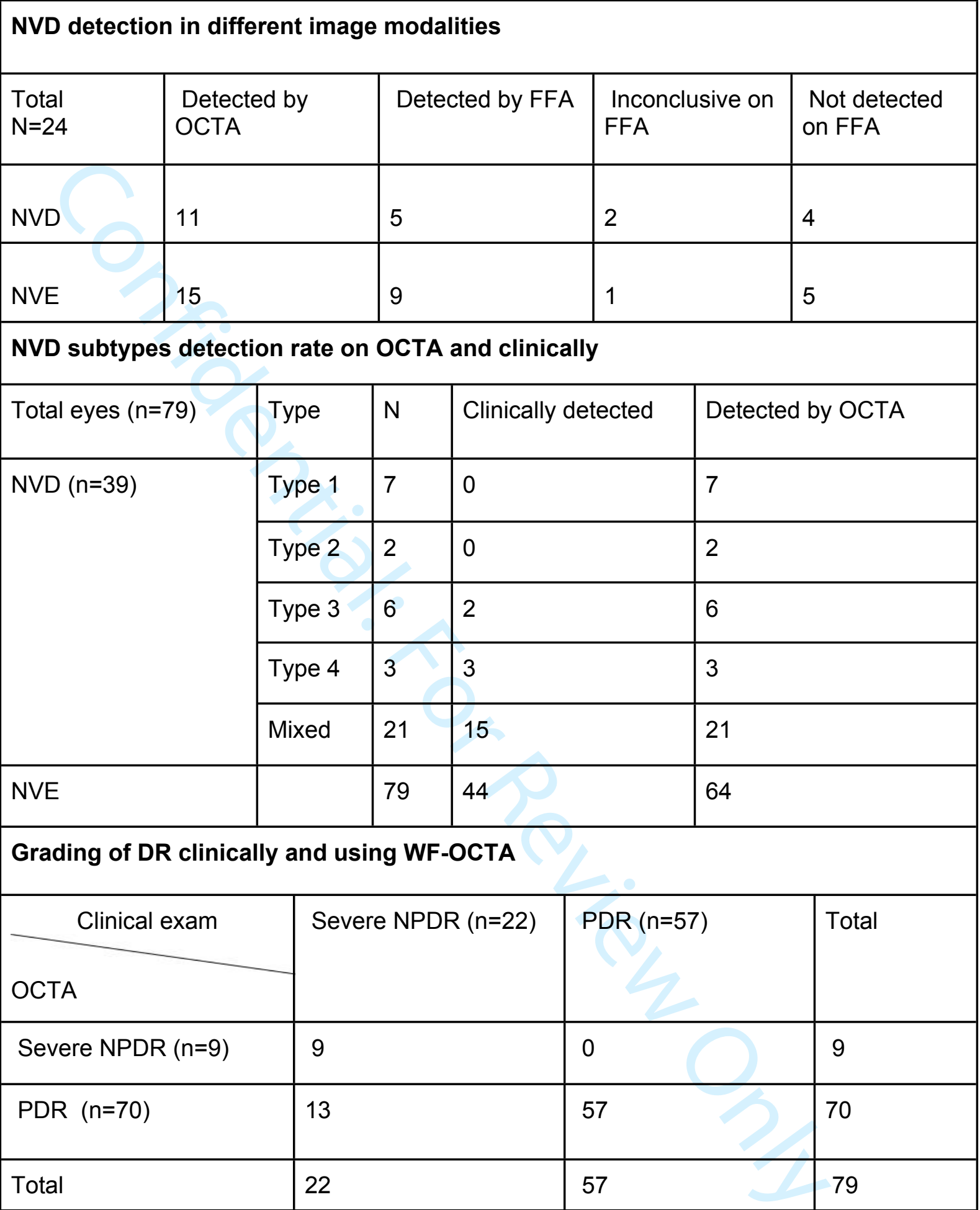

NVD: Neovascularisation of the disc NVE: Neovascularisation Elsewhere OCTA: optical coherence tomography angiography WF-OCTA: Wide-field optical coherence tomography angiography FFA: fundus fluorescein angiography NPDR: non proliferative diabetic retinopathy PDR: proliferative diabetic retinopathy 
Figure 1: a) UltraWide field fluorescein angiography (UWF-FFA) with overlying dotted blue square of the corresponding wide-field optical coherence tomography angiography (WF-OCTA) field, illustrating that despite the wider field of UWF-FFA, most of clinically significant data was detected within the WF-OCTA field. Additionally, the peripheral field in UWF-FFA shows artifacts that affect image interpretation. b) widefield OCTA montage with overlaid squares demonstrating the different retinal sectors imaged by the 5 (12x12) data cubes; Central, superonasal, superotemporal, inferonasal, and inferotemporal cubes.

$88 \times 42 \mathrm{~mm}(300 \times 300 \mathrm{DPI})$ 


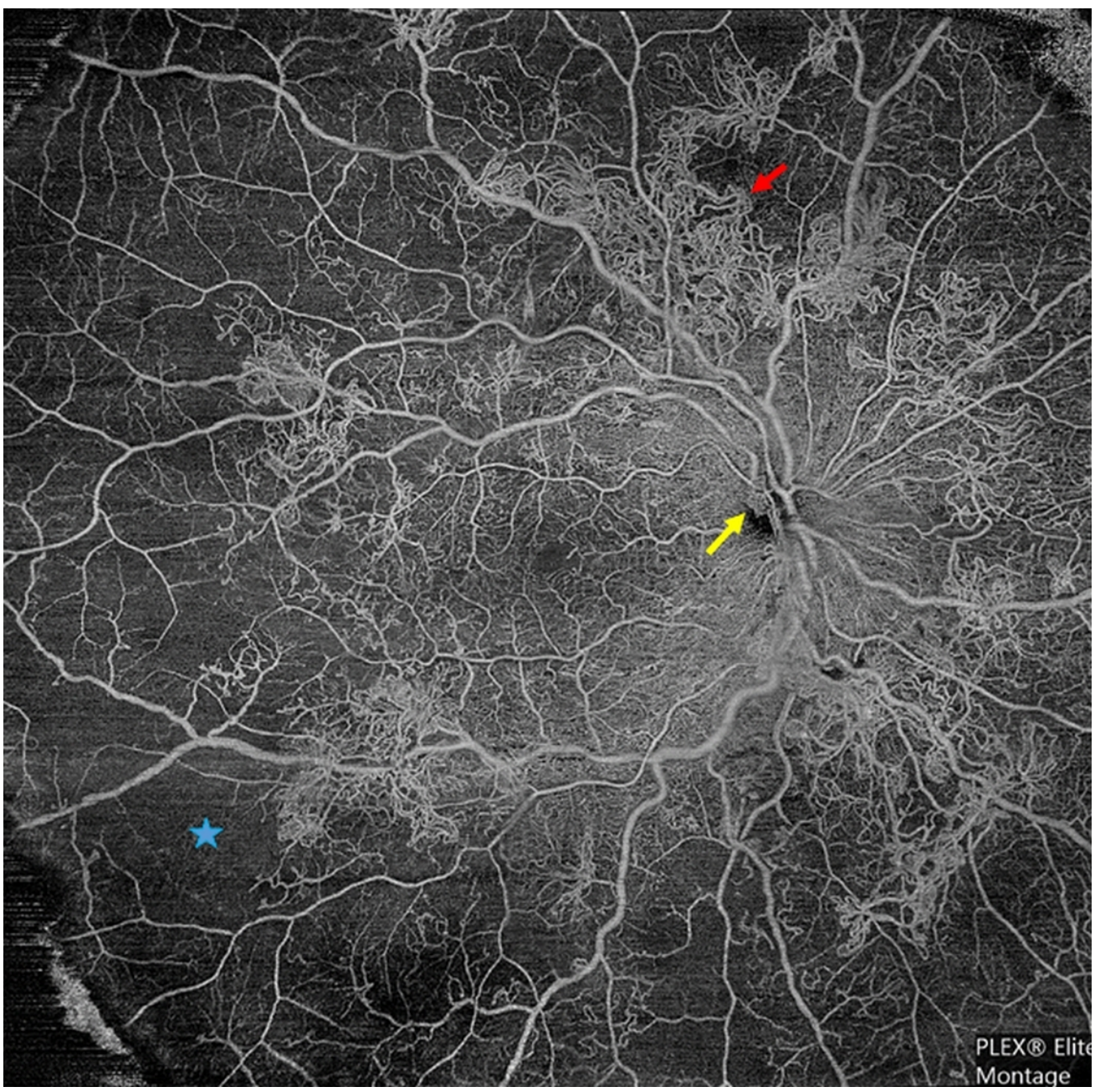

Figure 2: Wide-field en face optical coherence tomography angiography (WF-OCTA) montage at the level of the superficial capillary plexus slab showing multiple neovascularisation elsewhere (NVEs) mid peripherally (red arrow) at the edge of the ischaemic areas (star). Neovascularization of the disc (NVD) could also be detected (yellow arrow).

$88 \times 87 \mathrm{~mm}(300 \times 300 \mathrm{DPI})$ 


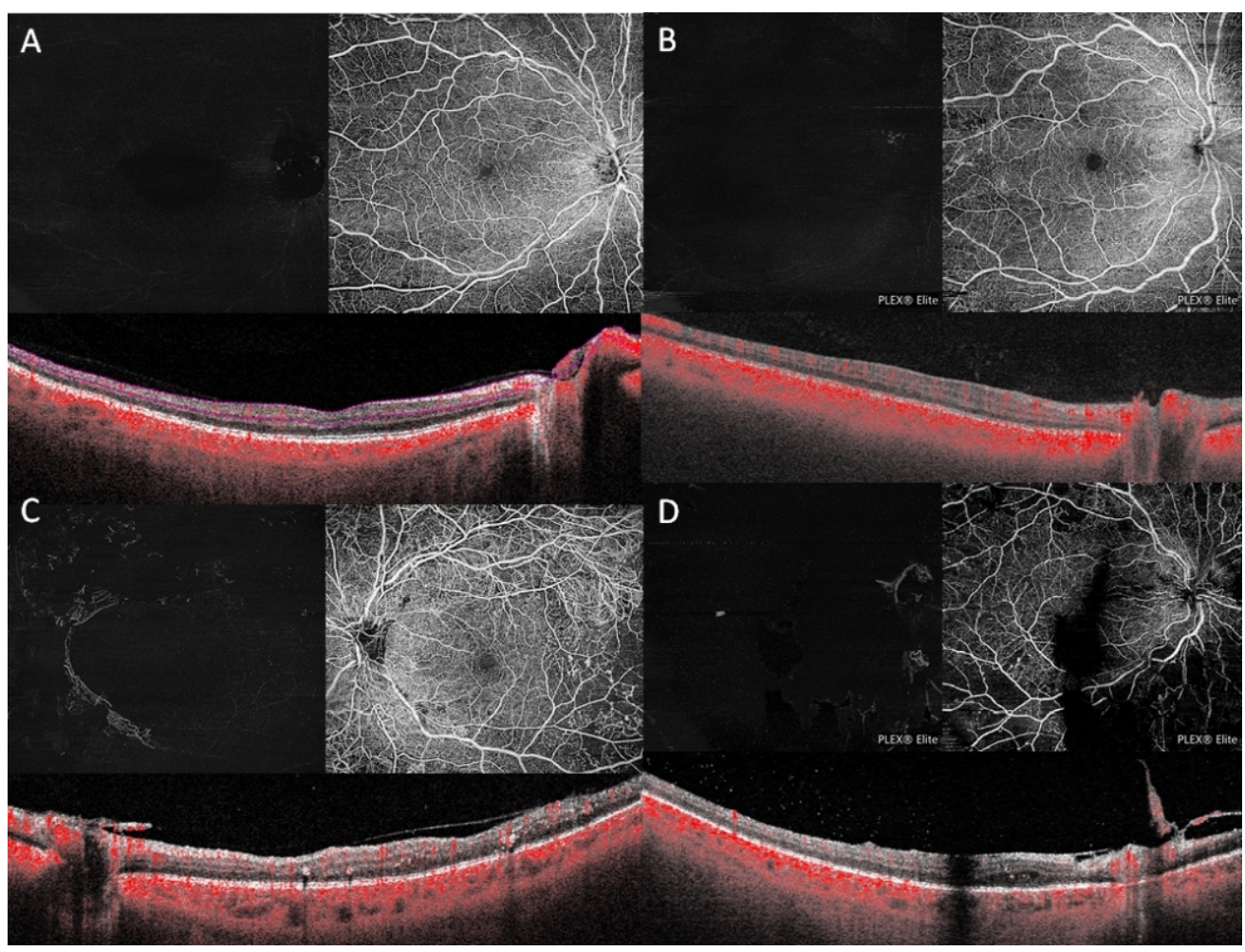

Figure 3: En face optical coherence tomography angiography (OCTA) at the superficial and vitreoretinal interface (VRI) slabs and the corresponding OCTA B-scans to illustrate the different types of neovascularization of the disc (NVD): a) Type 1 NVD: OCTA B-scan demonstrating demonstrating a preretinal hyperreflective material (PRHM) bridging the physiological cup with evident flow signal which could be seen at the superficial slab as it encompasses the physiological cup within. b) Type 2 NVD: which appear as small buds in the B- scan which could be seen at the VRI slab. c)Type 3 NVD: where the PRHM can be seen in the B-scan flat over the surface. This type could be detected in the VRI slab. d)Type 4 NVD: which is elevated above the surface as seen in the B-scan. This type is detectable at the VRI slab.

$88 \times 67 \mathrm{~mm}(300 \times 300 \mathrm{DPI})$ 


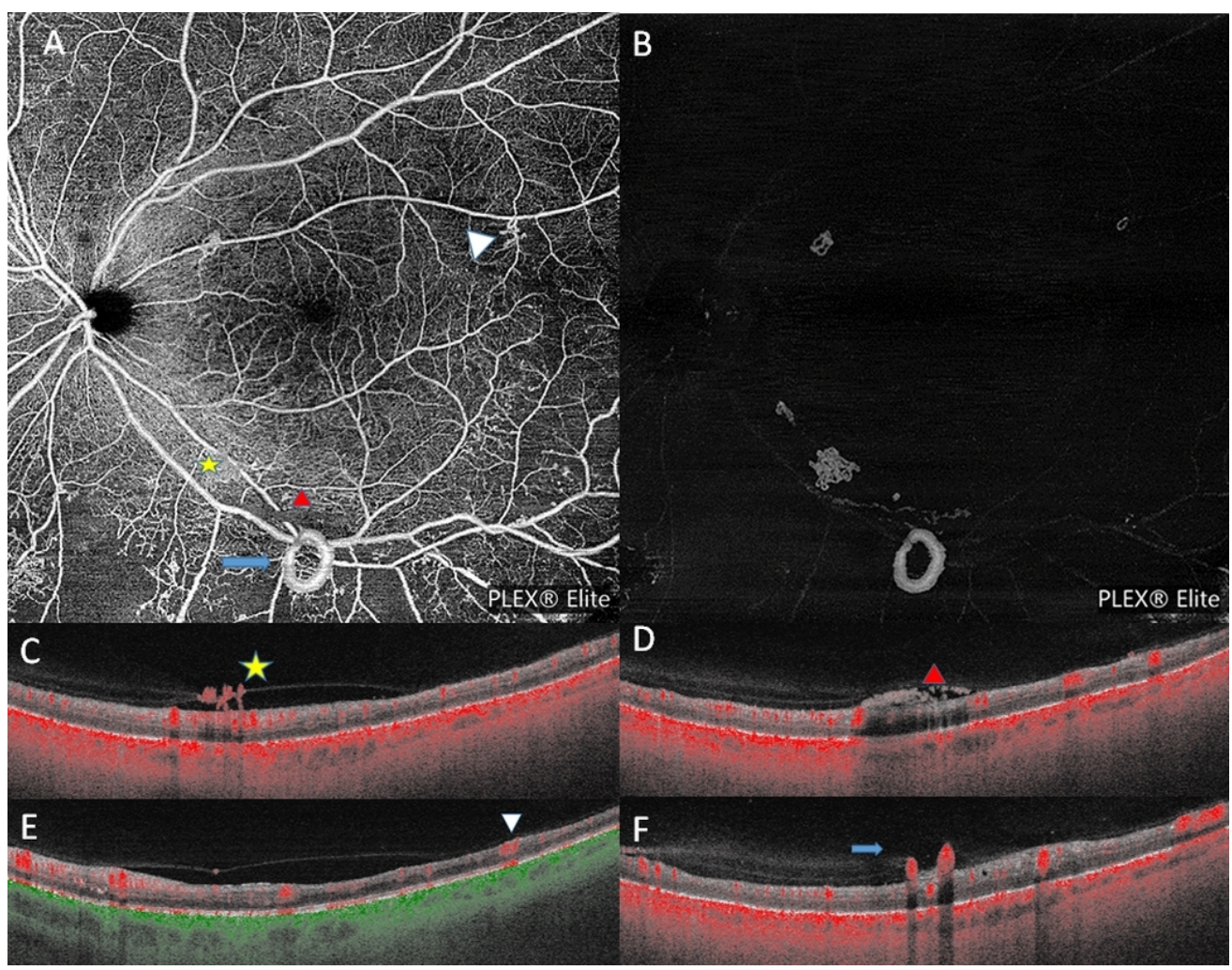

Figure 4: Central 12x12 enface optical coherence tomography Angiography (OCTA) at the superficial (A) and vitreoretinal interface (VRI) (B) slabs with multiple OCTA B-scans at different levels illustrating various diabetic retinopathy signs.C) Neovascularisation elsewhere (NVE) could be prominently seen as a preretinal hyperreflective material (PRHM) in the B-scan with evident flow signals which could be detected as network at the VRI level (yellow star), while intraretinal microvascular abnormalities (IRMA) (white arrow head) could be seen on the enface OCTA at the superficial slab as abnormal dilated blood vessels without any evident PRHM on the B-scan (E). OCTA may be helpful in differentiating PRHM that is secondary to preretinal haemorrhage (red arrow head) from an NVE (yellow star). Preretinal haemorrhage characterised by the absence of flow signals on OCTA B-scan along with backshadowing (D) OCTA can detect vascular loops as well (blue arrow) (F).

$88 \times 69 \mathrm{~mm}(300 \times 300 \mathrm{DPI})$ 


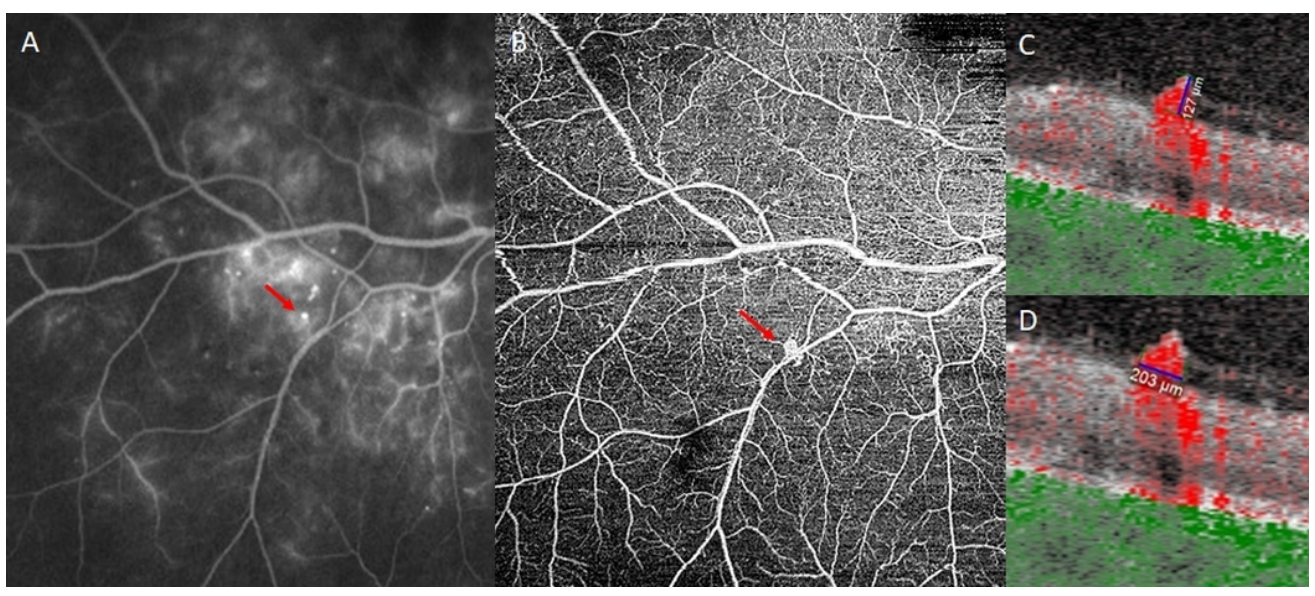

Figure 5: a) Late phase fluorescein angiography of the Inferotemporal quadrant showing small hyperfluorescent lesion (red arrow) that were diagnosed as microaneurysms. However, the corresponding enface OCTA (b) illustrated the presence of a small NVE (red arrow) over the infero-temporal arcade corresponding to preretinal hyperreflective material (PRHM) with a flow signal on OCTA B-scan (with vertical diameter of $127 \mu \mathrm{m}$ and horizontal diameter of $203 \mu \mathrm{m}$ ).

$88 \times 39 \mathrm{~mm}(300 \times 300 \mathrm{DPI})$ 